\title{
A Multifactorial Approach to Sleep and Its Association with Health-Related Quality of Life in a Multiethnic Asian Working Population: A Cross-Sectional Analysis
}

\author{
Gerard Dunleavy ${ }^{1, * \mathbb{C}}$, André Comiran Tonon 2,3® ${ }^{\mathbb{D}}$, Ai Ping Chua ${ }^{4}$, Yichi Zhang ${ }^{1}$, \\ Kei Long Cheung ${ }^{5}$, Thuan-Quoc Thach ${ }^{1}$, Yuri Rykov ${ }^{1}$, Chee-Kiong Soh $\left.{ }^{6}{ }^{(}\right)$, \\ Georgios Christopoulos ${ }^{7}$, Hein de Vries ${ }^{8}$ and Josip Car ${ }^{1,9}$ \\ 1 Centre for Population Health Sciences, Lee Kong Chian School of Medicine, Nanyang Technological \\ University Singapore, 11 Mandalay Road, Singapore 308232, Singapore; yczhang@ntu.edu.sg (Y.Z.); \\ thuanquoc.thach@ntu.edu.sg (T.-Q.T.); yuri.rykov@ntu.edu.sg (Y.R.); josip.car@imperial.ac.uk (J.C.) \\ 2 Laboratório de Cronobiologia e Sono, Porto Alegre Clínicas Hospital (HCPA), Porto Alegre 90035-007, Brazil; \\ andrectonon@gmail.com \\ 3 Postgraduate Program in Psychiatry and Behavioral Sciences, Federal University of Rio Grande Do \\ Sul (UFRGS), Porto Alegre 90040-060, Brazil \\ 4 Department of Medicine, Jurong Health Campus, National University Health System, 1 Jurong East Street 21, \\ Singapore 609606, Singapore; ai_ping_chua@nuhs.edu.sg \\ 5 Department of Clinical Sciences, College of Health and Life Sciences, Brunel University London, \\ London UB8 3PH, UK; keilong.cheung@brunel.ac.uk \\ 6 School of Civil and Environmental Engineering, College of Engineering, Nanyang Technological University \\ Singapore, 50 Nanyang Avenue, Singapore 639798, Singapore; csohck@ntu.edu.sg \\ 7 Division of Leadership, Management and Organisation, Nanyang Business School, College of Business, \\ Nanyang Technological University Singapore, 50 Nanyang Avenue, Singapore 639798, Singapore; \\ cgeorgios@ntu.edu.sg \\ 8 Department of Health Promotion, CAPHRI Care and Public Health Research Institute, Maastricht University, \\ Maastricht POB 6166022 MD, The Netherlands; hein.devries@maastrichtuniversity.nl \\ 9 Department of Primary Care and Public Health, School of Public Health, Imperial College London, \\ London SW7 2AZ, UK \\ * Correspondence: gerard.dunleavyhp@gmail.com; Tel.: +65-6904-7021
}

Received: 19 August 2019; Accepted: 23 October 2019; Published: 28 October 2019

\begin{abstract}
This study aims to explore if objectively and subjectively measured sleep parameters are associated with physical and mental health-related quality of life in a multiethnic working population in Singapore. We performed a cross-sectional analysis with data from 329 full-time employees enrolled in a workplace cohort study in Singapore. The Short-Form 36v2 (SF-36v2) survey was used to assess health-related quality of life, in terms of physical and mental health. Subjective and objective sleep parameters were measured using the Pittsburgh Sleep Quality Index and wrist actigraphy, respectively. Generalized linear modeling was performed to examine the association between sleep parameters and health-related quality of life. After adjusting for confounders, subjectively measured sleep disturbances were associated with a lower physical health-related quality of life, whereas higher, objectively measured sleep efficiency was associated with greater physical health-related quality of life. Subjectively measured daytime dysfunction was associated with impaired mental health-related quality of life. Using both objective and subjective measurements of sleep, the current study suggests that there is an association between sleep and health-related quality of life. Workplace health-promotion planners in Singapore should consider programmes that educate workers on better sleep hygiene practices in an effort to improve sleep and health-related quality of life.
\end{abstract}


Keywords: sleep quality; quality of life; workplace health

\section{Introduction}

The sleep-wake cycle is a complex phenomenon that encompasses several physiological and behavioral oscillations [1]. Although no consistent evidence has proven the reasons why humans sleep [2], a growing body of literature shows that low sleep quantity or poor sleep quality is associated with hypertension, heart disease, stroke, diabetes, and even cancer, as well as mood disorders, commonly anxiety and depression [3,4]. Other than physical and mental ailments, symptoms of sleepiness and fatigue from sleep deficiency during wake periods can lead to impaired performance, reduced work productivity, increased workplace absenteeism, and risk of work-related accidents [3]. In a recent study, residents of Singapore, a city-state that is heavily reliant on its human capital, were reported to have the shortest sleep duration of the 20 countries enrolled in the study [5]. Therefore, the negative effects of sleep deprivation and poor sleep quality are of concern in Singapore. The lack of sleep among residents of Singapore is often linked to the well-established culture of working long hours. Full-time workers in Singapore worked an average of $45 \mathrm{~h}$ per week in 2019 [6]. In contrast, the average full-time worker in the U.S. and the EU worker spent $35 \mathrm{~h}$ and $42 \mathrm{~h}$ working per week, respectively [7]. Another challenge to both sleep quality and duration for residents of Singapore is the excessively floodlit society in which they live as the city-state is reported to be the most light-polluted country in the world [8]. Given the prevalence of poor sleep quality, daytime fatigue, and short sleep duration of residents in Singapore [5,9], research is needed to examine how sleep impacts the health-related quality of life of working populations in Singapore.

Quality of life is a multidimensional concept encompassing physical, psychological, and social domains, all of which are shown to be negatively impacted by reduced sleep quantity or poor sleep quality [10]. The 36 Item Short Form Health Survey questionnaire (SF-36) is a generic instrument that has been used to measure, assess, and evaluate the quality of life of different populations in Singapore [11-13]. The SF-36 assessment produces two summary measurements of health-related quality of life, namely, a physical component summary score which represents physical health-related quality of life, and a mental component summary score which represents mental health-related quality of life. Considering the multifaceted nature of sleep, there is no consensus on what is the best variable to measure and report for the study of physical and mental health-related quality of life. Common direct subjective measurements of sleep are self-reported sleep quality and satisfaction, average sleep duration, and sleep efficiency (sleep latency and wake after sleep onset). Indirect subjective measurements include self-perceived sleepiness and unintentional napping during awake working periods, as well as work and academic performance. The Pittsburgh Sleep Quality Index (PSQI) which was introduced in 1989 has remained one of the most popular, well-established and validated clinical tools used to collect both direct and indirect measurements of subjective sleep quality [14], albeit its limitations. Direct objective measurements of sleep duration and quality are actigraphy and polysomnogram (PSG), and indirect measurements are mean sleep latency test, mean wakefulness test, and various forms of vigilance performance testings. Actigraphy has emerged as an attractive, practical and cheaper alternative to PSG, which has been extensively validated. Unlike PSG, it allows for prolonged continuous periods of sleep evaluation in the subject's natural work and sleep environments without being labor-intensive $[15,16]$. A number of studies have reported on the association between subjectively assessed parameters of sleep and health-related quality of life [17-25], while fewer have examined their relationship with objective measurements of sleep [26]. Given the difference in cost, accessibility, and ease of administration, it is understandable that subjective assessments of sleep are often opted for over objective measurements, however, the extant literature has demonstrated inconsistencies between subjectively and objectively measured sleep [27-29], and therefore studies that include both methods are warranted. A further gap that exists in the literature is the lack of 
studies that examine the relationship between sleep and health-related quality of life in working populations, with the majority of studies that investigate this relationship conducted among specific clinical populations [23,30-33]. To our knowledge, no study has investigated whether sleep is associated with physical and mental health-related quality of life in a working population in Singapore.

The goal of this study is to explore if sleep parameters are associated with physical and mental health-related quality of life in a multiethnic working population in Singapore. We aim to describe the independent effects of subjective sleep parameters and objective actigraphy-based data, accounting for confounding factors (i.e., sociodemographic characteristics, lifestyle, and occupational factors).

\section{Materials and Methods}

\subsection{Study Design and Sample}

We conducted a cross-sectional analysis of data of 329 adults (21 years and above) who were participating in a workplace cohort study in Singapore. Details of the cohort study design are published elsewhere [34]. Briefly, a cohort of 464 full-time employees was set up in four workplaces in Singapore to study the effects of working underground vs. aboveground on sleep quality and melatonin levels. The four workplaces included companies from the transport industry, cooling plants, and universities. Participants from these workplaces were invited to participate in the study via meetings, workplace posters, and emails. Those willing to participate were then screened for eligibility using the following criteria: aged 21 years and above, should be working for at least four hours per day, not pregnant, and had not traveled overseas across a different time zone within the past six months.

\subsection{Measurements}

\subsubsection{Health-Related Quality of Life}

The Short Form 36 (SF-36v2) survey form was used to assess the health-related quality of life (HRQoL) of participants [35]. The SF-36v2 dimensions are divided into the following two categories: physical component summary (PCS) and mental component summary (MCS), which represent physical and mental health-related quality of life, respectively, and will be referred to as such hereafter in this article. Physical health-related quality of life has 21 items and measures the following four domains: physical functioning (10 items, the following items are about activities you might do during a typical day, example question, "Does your health now limit you in these activities? If so, how much? Lifting or carrying groceries?" Yes, limited a lot, yes, limited a little, no, not limited at all); role limitations due to physical health ( 4 items, example question, "During the past 4 weeks, have you cut down the amount of time you spent on work or other activities as a result of your physical health?" yes or no); bodily pain ( 2 items, example question, "During the past 4 weeks, how much did pain interfere with your normal work (including both work outside the home and housework)?" not at all, a little bit, moderately, quite a bit, extremely); and general health perception (5 items, example question, "I am as healthy as anybody I know." definitely true, mostly true, don't know, mostly false, or definitely false). Mental health-related quality of life contains 14 items and measures the following four domains: social functioning ( 2 items, example question, "During the past 4 weeks, how much of the time has your physical health or emotional problems interfered with your social activities (like visiting with friends, relatives, etc.)?" not at all, slightly, moderately, severe or very severe); role limitations due to emotional problems ( 3 items, example question, "During the past 4 weeks, have you accomplished less than you would like as a result of any emotional problems (such as feeling depressed or anxious)?" yes or no); vitality (4 items, example question, "During the past 4 weeks, did you have a lot of energy?" all of the time, most of the time, a good bit of the time, some of the time, a little bit of the time or none of the time); and emotional well-being ( 5 items, example question, "During the past 4 weeks, have you been a very nervous person?" all of the time, most of the time, a good bit of the time, some of the time, a little bit of the time or none of the time). The scoring of the two summary components was performed using 
the Health Outcomes Scoring Software 5.1 (QualityMetric Health Outcomes ${ }^{\mathrm{TM}}$, Lincoln, UK) based on 2009 U.S. norms with a mean of 50 and a standard deviation of 10. Higher scores indicate a better health-related quality of life.

\subsubsection{Sleep Parameters}

\section{Subjective Measurements}

The Pittsburgh Sleep Quality Index (PSQI) measures sleep quality during the past month [14]. It has 19 self-rated items, which are grouped into seven subscales: subjective sleep quality (1 item), sleep latency ( 2 items), sleep duration ( 1 item), habitual sleep efficiency ( 3 items), sleep disturbances (9 items), use of sleeping medications ( 1 item), and daytime dysfunction ( 2 items). Subjective sleep quality was assessed with the PSQI item "During the past month, how would you rate your sleep quality overall?". The sleep latency subscale examines how long the participant takes to fall asleep, while the sleep duration subscale examines how many hours of actual sleep the participant gets at night. Habitual sleep efficiency was calculated as follows: hours of sleep/(get up time - usual bedtime) $\times 100$. Sleep disturbance was calculated from the PSQI items "wake up in the middle of the night or early morning", "need to get up to use the bathroom", "cannot breathe comfortably", "cough or snore loudly", "feel too cold", "feel too hot", "have bad dreams", and "have pain". Daytime dysfunction was calculated from the PSQI items, "During the past month, how often have you taken medicine to help you sleep (prescribed or over the counter)?" and "During the past month, how often you have you had trouble staying awake while driving, eating meals, or engaging in social activity?". The score for each component ranges from 0 to 3 , with higher scores indicating greater difficulty. The sum of scores for these seven components provides a global score (ranges from 0 to 21), with " 0 " indicating no difficulty and " 21 " indicating severe difficulties in all areas. Participants with a PSQI global score $>5$ were categorized as having poor sleep quality, as per the recommended cut-off by the developers of the screening tool and subsequent validation studies of the instrument [14,36]. Standard scoring of the PSQI was applied.

\section{Objective Sleep Measurements}

Participants also wore an Actiwatch (Actiwatch Spectrum Plus, Phillips Respironics), which contains an accelerometer capable of estimating locomotor activity (e.g., movement, rest and activity periods). Participants were requested to wear the Actiwatch on their non-dominant wrist, $24 \mathrm{~h}$ a day, for a period of eight consecutive days. The participants were instructed on how to use the device by trained staff and they were also requested to complete a sleep diary on a daily basis. Each actigraph was evaluated by a scorer who used a standardized approach to set rest intervals (periods when the subject was trying to sleep) based on 3 inputs: event markers, sleep diary, white light intensity and activity. Actigraphy data were aggregated in $30 \mathrm{~s}$ epochs and scored manually as sleep or wake using a procedure published by the NSRR and based on (a) an event marker on the ActiWatch device, (b) the sleep diary, and (c) activity and ambient light data from the ActiWatch device [37]. Participants were instructed to press the event marker (a button on the side of the ActiWatch device) when going to sleep for the evening and when awoken in the morning. The actigraphy variables being reported herein included: (1) total sleep time (hours), which reflects the sum of those epochs between "sleep start" and "sleep end" that were designated as "sleeping"; (2) sleep efficiency (percent \%), which is an index of the amount of time in bed actually spent sleeping, is determined by dividing total-sleep-time by the time-in-bed, multiplied by 100; and (3) wake after sleep onset (WASO), which refers to periods (minutes) of wakefulness occurring after sleep onset. 


\subsubsection{Confounding Factors}

Health and Lifestyle Factors

Smoking status of participants was classified as a non-smoker, ex-smoker, and current smoker. Alcohol drinking status was defined as non-drinker, drinks less than once a month, and drinks once or more than once a month. Physical activity was measured using the Global Physical Activity Questionnaire, which measures activity levels in three domains, namely, work, travel, and leisure. A metabolic equivalent (MET) value of four was assigned for moderate physical activities and a MET value of eight for vigorous physical activities. The duration (in minutes) of an activity performed in each of the three domains was multiplied by its MET value, and these were summed to obtain the total MET-min/week. Individuals with a total MET-min/week $<600,600-2999$, and $\geq 3000$ were considered to be less active, moderately active, and highly active, respectively. Body mass index (BMI) was calculated as weight in kilograms $(\mathrm{kg}$ ) divided by height in meters $(\mathrm{m})$ squared. Self-reported chronic conditions were assessed using questions on the history of various chronic medical conditions including diabetes, heart disease, stroke, high cholesterol, hypertension, chronic kidney disease, peripheral vascular disease, asthma, allergy, and mental disorders. Stress at home, stress at work, and financial stress were each assessed with single-item questions [38].

\section{Occupational Factors}

Participants were asked about their work location (underground or aboveground); shift work (yes or no); job type (office, control room, and workshop); number of work hours per week; and number of years working for the present company.

\subsection{Statistical Analysis}

Baseline characteristics of participants and the distribution of objective and subjective sleep parameters are summarized using mean (SD: standard deviation) or median (IQR: inter-quartile range) for continuous variables and using frequency and percentage for categorical variables. This study has two dependent variables, physical and mental health-related quality of life. To assess whether various sleep parameters were independently associated with health-related quality of life, we fitted four generalized linear models (GLM) with a Gaussian distribution family using a robust standard error in the following hierarchical fashion, adjusting for sociodemographic characteristics, health and lifestyle factors, and occupational factors. For the first model examining physical health-related quality of life, confounding factors were added in a hierarchical fashion and then the objective sleep parameters were subsequently addedto the final model. For the second model examining physical health-related quality of life, confounding factors were similarly added and then subjective sleep subscales were subsequently included. The same modelling approach was applied to examine mental health-related quality of life with separate analyses performed with subjective and objective sleep parameters. Results for the GLM analysis were expressed as adjusted regression coefficients with $95 \%$ confidence intervals using robust standard errors. A negative coefficient reflects a decrease in physical and mental health-related quality of life and a positive coefficient indicates an increase. A two-tailed $p$-value $<0.05$ was considered to be statistically significant. Pearson's correlations were performed to determine the strength of the relationship between subjective and objective sleep parameters. Objective and subjectively measured sleep duration were compared, as were objectively and subjectively measured sleep efficiency. Objectively measured wake after sleep onset was considered a proxy for sleep disturbances, thus the correlation of these variables was examined. All statistical analyses were conducted using Stata 15.0 software (StataCorp, College Station, TX, USA).

\subsection{Ethics Approval}

The study was conducted in accordance with the Declaration of Helsinki and approved by the Institutional Review Board of Nanyang Technological University Singapore, Singapore 
(IRB-2015-11-028). Written informed consent was obtained from all study participants prior to the commencement of data collection.

\section{Results}

\subsection{Characteristics of Study Participants}

We studied 329 working adults in Singapore. The sociodemographic characteristics of the study population are shown in Table 1. The age of the sample ranged between 22 and 65 years with a mean age of 40.7 (SD:11.1) years, and 78.1\% were male. Reflecting national representation, a large proportion were Chinese (65.7\%), followed by Malays (18.2\%), Indians (11.9\%), and other Asian groups (4.3\%). The majority of participants were married $(64.7 \%)$, had at least postsecondary education $(89.7 \%)$, and were earning a monthly income $<\$ \$ 4,000(69.0 \%)$.

Table 1. Characteristics of study participants and physical component summary (PCS) and mental component summary (MCS) scores.

\begin{tabular}{lccc}
\hline \multicolumn{1}{c}{ Demographics } & Total & PCS (Mean \pm SD) & MCS (Mean \pm SD) \\
\hline Age (years) & $40.7 \pm 11.1$ & & \\
$21-30$ & $84(28.0)$ & $52.1 \pm 5.5$ & $48.8 \pm 7.2$ \\
$31-40$ & $98(28.9)$ & $50.8 \pm 6.9$ & $49.1 \pm 7.0$ \\
$40+$ & $147(43.2)$ & $51.2 \pm 6.1$ & $51.6 \pm 7.9$ \\
\hline Gender & & & \\
Male & $256(77.8)$ & $51.3 \pm 6.4$ & $49.7 \pm 7.6$ \\
Female & $73(22.2)$ & $51.5 \pm 5.5$ & $51.7 \pm 7.1$ \\
\hline Education & & & $51.4 \pm 9.1$ \\
Primary and secondary & $32(10.3)$ & $52.0 \pm 5.0$ & $50.3 \pm 7.6$ \\
Pre-college & $178(54.1)$ & $50.2 \pm 6.4$ & $49.5 \pm 7.0$ \\
College and above & $119(35.6)$ & $52.9 \pm 5.8$ & $50.2 \pm 7.7$ \\
\hline Ethnicity & & & $49.9 \pm 8.0$ \\
Chinese & $216(65.7)$ & $52.0 \pm 5.9$ & $49.4 \pm 5.7$ \\
Malay & $60(18.2)$ & $49.3 \pm 6.7$ & $52.5 \pm 9.0$ \\
Indian & $39(11.9)$ & $50.1 \pm 6.8$ & \\
Others ${ }^{\text {a }}$ & $14(4.3)$ & $53.0 \pm 5.1$ & $50.2 \pm 7.3$ \\
\hline Marital status & & $50.1 \pm 7.7$ \\
Single ${ }^{\text {b }}$ & $116(35.3)$ & $52.3 \pm 5.8$ & $49.9 \pm 7.9$ \\
Married & $213(64.7)$ & $50.8 \pm 6.3$ & $50.7 \pm 6.9$ \\
\hline Monthly income & & & \\
$<$ S $\$ 4,000$ & $227(69.0)$ & $51.2 \pm 6.0$ & \\
$\geq$ S $\$ 4,000$ & $102(31.0)$ & $51.7 \pm 6.5$ & \\
\hline Continus & & & \\
\hline
\end{tabular}

Continuous variables are presented as mean \pm standard deviation, and categorical variables as $\mathrm{n}(\%) ;{ }^{\mathrm{a}}$ includes mixed ethnicities, Indonesians, Pakistanis and Filipinos; ${ }^{b}$ includes never married, widowed, and divorced; SD, standard deviation.

The overall PCS and MCS scores for the sample were 51.3 (SD: 6.2) and 50.2 (SD: 7.5), respectively. Table 2 shows the objectively and subjectively measured sleep parameters of the study population. According to study participants objective sleep measurements, participants slept an average of 5.8 (SD: 0.9) hours per night, were awake after sleep onset for an average of 37.9 (IQR: 28.1-49.5) minutes per night, and had a sleep efficiency of 79.1\% (SD: 7.5). The mean PSQI global score was 5.1 (SD: 2.8). The results of the univariate analysis for PCS and MCS are provided in the Supplementary Materials (See Table S2). 


\subsection{Relationship between Objective and Subjective Measurements of Sleep}

Subjective and objective sleep measurements were either weakly or not significantly correlated. There was a small but significant correlation between objectively and subjectively measured sleep duration $(r=0.27, p<001)$, while there was no association between objectively and subjectively measured sleep efficiency. There was no association between objectively measured wake after sleep onset and subjectively assessed sleep disturbances.

Table 2. Distribution of objective and subjective sleep parameters

\begin{tabular}{lc}
\hline \multicolumn{1}{c}{ Sleep Parameters } & Total \\
\hline Objective sleep parameters & \\
Total sleep time (hours), mean (SD) & $5.8 \pm 0.9$ \\
$<5 \mathrm{~h}$ & $53(19.0)$ \\
$5-6 \mathrm{~h}$ & $111(40.0)$ \\
$6-7 \mathrm{~h}$ & $97(34.8)$ \\
$>7 \mathrm{~h}$ & $18(6.5)$ \\
Wake after sleep onset (min), median (IQR) & $37.9(28.1-49.5)$ \\
Sleep efficiency (\%) & $80.9 \pm 7.5$ \\
\hline Subjective sleep parameters & \\
PSQI global score & $5.1 \pm 2.8$ \\
Subjective sleep quality, median (IQR) & $1(0-2)$ \\
Sleep latency, median (IQR) & $1(0-1)$ \\
Sleep duration, median (IQR) & $1(0-2)$ \\
Habitual sleep efficiency, median (IQR) & $0(0-1)$ \\
Sleep disturbances, median (IQR) & $1(1-1)$ \\
Use of sleeping medications, median (IQR) & $0(0-0)$ \\
Daytime dysfunction, median (IQR) & $1(0-1)$ \\
\hline \multicolumn{2}{c}{ IQR, inter-quartile range; SD, standard deviation. }
\end{tabular}

\subsection{Association between Sleep Parameters and Physical Health-Related Quality of Life}

Table 3 shows the association between the various sleep parameters and physical health-related quality of life. In unadjusted models, higher scores (indicating a worse sleep quality), in five of the seven of the subjectively assessed domains of sleep (subjective sleep quality, sleep latency, sleep duration, sleep disturbances, and daytime dysfunction) were associated with impaired physical health-related quality of life. In fully adjusted models, subjectively assessed sleep disturbances were associated with impaired physical health-related quality of life, while higher, objectively measured, sleep efficiency was associated with greater physical health-related quality of life. The results of the multivariate analysis for objective and subjective sleep parameters and physical health-related quality of life are provided in the Supplementary Materials (See Tables S3 and S4).

\subsection{Association between Sleep Parameters and Mental Health-Related Quality of Life}

Table 4 shows the association between the various sleep parameters and mental health-related quality of life. In unadjusted models, higher scores (indicating a worse sleep quality), in five of the seven of the subjectively assessed domains of sleep (subjective sleep quality, sleep latency, sleep duration, sleep disturbances, and daytime dysfunction) were associated with impaired mental health-related quality of life. In fully adjusted models, a higher score for subjectively assessed daytime dysfunction was associated with impaired mental health-related quality of life. The results of the multivariate analysis for objective and subjective sleep parameters and mental health-related quality of life are provided in the Supplementary Materials (See Tables S5 and S6). 
Table 3. Association between objective and subjective sleep parameters on physical health-related quality of life.

\begin{tabular}{|c|c|c|}
\hline Sleep Parameters & Standardized $\beta(95 \%$ CI) & Standardized $\beta_{\mathrm{adj}}(95 \% \mathrm{CI})^{\wedge}$ \\
\hline \multicolumn{3}{|l|}{ Subjective sleep parameters } \\
\hline Subjective sleep quality & $-2.720(-3.835,-1.605) * * *$ & $-1.416(-2.846,0.014)$ \\
\hline Sleep latency & $-1.771(-2.518,-1.024)^{* * *}$ & $-0.704(-1.522,0.114)$ \\
\hline Sleep duration & $-1.364(-2.067,-0.661) * * *$ & $-0.416(-1.220,0.387)$ \\
\hline Habitual sleep efficiency & $-0.072(-0.912,0.768)$ & $0.697(-0.118,1.512)$ \\
\hline Sleep disturbances & $-3.430(-4.598,-2.262)^{* * *}$ & $-1.829(-3.216,-0.443) *$ \\
\hline Use of sleeping medications & $-1.294(-2.883,0.295)$ & $-0.593(-2.052,0.866)$ \\
\hline Daytime dysfunction & $-1.861(-2.835,-0.886)^{* * *}$ & $-0.909(-1.948,0.130)$ \\
\hline \multicolumn{3}{|l|}{ Objective sleep parameters } \\
\hline Total sleep time (hours) & $0.266(-0.559,1.090)$ & $0.777(-1.802,0.248)$ \\
\hline Wake after sleep onset (min) & $0.002(-0.041,0.045)$ & $0.049(-0.007,0.106)$ \\
\hline Sleep efficiency $(\%)$ & $0.125(0.030,0.220)^{*}$ & $0.211(0.050,0.372)^{*}$ \\
\hline
\end{tabular}

Table 4. Association between objective and subjective sleep parameters on mental health-related quality of life.

\begin{tabular}{|c|c|c|}
\hline Sleep Parameters & Standardized $\beta(95 \% \mathrm{CI})^{\wedge}$ & Standardized $\beta_{\text {adj }}(95 \% \mathrm{CI})^{\wedge}$ \\
\hline \multicolumn{3}{|l|}{ Subjective sleep parameters } \\
\hline Subjective sleep quality & $-4.726(-6.037,-3.415)^{* * *}$ & $-1.415(-2.939,0.110)$ \\
\hline Sleep latency & $-2.203(-3.114,-1.293) * * *$ & $-0.809(-1.704,0.086)$ \\
\hline Sleep duration & $-1.282(-2.148,-0.416)^{* * *}$ & $-0.027(-0.891,0.838)$ \\
\hline Habitual sleep efficiency & $-0.224(-1.249,0.800)$ & $0.526(-0.341,1.394)$ \\
\hline Sleep disturbances & $-2.695(-4.163,-1.228)^{* * *}$ & $0.243(-1.168,1.653)$ \\
\hline Use of sleeping medications & $-1.434(-3.375,0.506)$ & $-0.722(-2.221,0.776)$ \\
\hline Daytime dysfunction & $-5.243(-6.316,-4.170) * * *$ & $-2.945(-4.136,-1.754) * * *$ \\
\hline \multicolumn{3}{|l|}{ Objective sleep parameters } \\
\hline Total sleep time (hours) & $-0.374(-1.373,0.626)$ & $-0.140(-1.376,1.096)$ \\
\hline Wake after sleep onset (mins) & $0.020(-0.032,0.071)$ & $0.052(-0.005,0.110)$ \\
\hline Sleep efficiency $(\%)$ & $0.019(-0.097,0.136)$ & $0.023(-0.131,0.177)$ \\
\hline
\end{tabular}

\section{Discussion}

\subsection{Main Findings}

The present study studied a non-clinical sample of 329 fulltime workers in Singapore to explore if objectively and subjectively measured sleep parameters were associated with physical and mental health-related quality of life. The findings confirmed that subjectively reported sleep disturbances were associated with a lower physical health-related quality of life. The findings also confirmed that higher sleep efficiency, objectively measured, was associated with greater physical health-related quality of life. The PSQI domain daytime dysfunction was associated with a lower mental health-related quality of life. The mean (SD) PSQI score in this study was 5.1 (SD: 2.8), which is in line with scores reported in the general population in Singapore [39], and significantly lower than what is observed from clinical populations and studies with older adults in Singapore [40,41].

\subsection{Evidence on the Impacts of Sleep in Physical and Mental Health-Related Quality of Life}

In our sample, we found a statistically significant association between sleep disturbances and physical health-related quality of life. This is not surprising as previous research has reported that physical health-related quality of life declines as sleep disturbances and insomnia become chronic [21,24]. The subscale sleep disturbances is quite broad in its composition and comprises many aspects of 
sleep quality. Each question that is used in the computation of the sleep disturbances subscale is aimed at eliciting possible underlying medical and physical conditions, sleep disorders, psychological or behavioural issues, environmental factors or a combination of any of these aspects, which can impact sleep adversely. Although a number of questions contributing towards the sleep disturbances subscale focuses on health problems that impact subjective sleep perception, it might be the case that the association of this subscale with physical health-related quality of life is mediated by underlying health conditions. Mental health-related quality of life is also reported to be impacted by sleep disturbances and insomnia, although only at the onset of sleep disturbances and insomnia with mental health-related quality of life stabilizing subsequently [24]. We also observed a significant association between sleep efficiency and physical health-related quality of life. Lower sleep efficiency indicates a higher sleep onset latency and more time awake after sleep onset, both of which are associated with anxiety-related insomnia, and lower sleep efficiency is also associated with sleep fragmentation clinical disorders, the most common being obstructive sleep apnea [42]. Lower sleep efficiency has also been found to be associated with metabolic syndrome [43] and all-cause mortality [44]. As reported in epidemiological literature, the effects of sleep not only impact our physical, but also our mental health $[17,25,45]$. The significant association between daytime dysfunction and mental health-related quality of life is not surprising as the daytime dysfunction subscale comprises of one question regarding fatigue and motivation and another question about trouble staying awake and daytime sleepiness, both of which may indicate depressive symptomatology. It is also well documented that individuals experiencing daytime dysfunction as a result of sleepiness are more likely to present depressive symptoms $[18,22,33,46]$. One cause of daytime dysfunction is insufficient sleep, and participants in our study averaged $5.8 \mathrm{~h}$ of sleep a night, more than an hour short of the 7 to $9 \mathrm{~h}$ recommended for optimal health [47]. It should also be acknowledged that there is sufficient evidence to suggest a relationship of bidirectionality in the association of impaired sleep and poor physical and mental health-related quality of life [48-51].

\subsection{The Application of Complimentary Sleep Measurements}

Our approach to examining sleep and health-related quality of life is novel in terms of applying both subjective and objective measurements of sleep to examine their relationship. Prior research has highlighted discrepancies between objective and subjective sleep measurements [28,52,53], and the use of both methods provides additional dimensions from which to approach and examine the relationship between sleep and health-related quality of life. Strengths and weaknesses are present in both objective and subjective measurements of sleep, sleep quantity determined by self-report questionnaires is unable to differentiate between time spent awake in bed and time asleep, whereas actigraphy data is unable to accurately identify if an individual suffers from sleep disturbances. The coupling of both methods captures different aspects of sleep and complement each other to provide a more complete and holistic picture of an individual's sleep. Furthermore, their combined use will very likely raise the chances of identifying people with problematic sleep practices or sleep disorders.

The application of subjective and objective sleep measurements in parallel to each other in this study identified associations between domains of health-related quality of life and subjectively measured sleep disturbances and daytime dysfunction, and objectively measured sleep efficiency. Higher objective, but not subjective, sleep efficiency was associated with greater physical health-related quality of life. This demonstrates the added benefit of a multifactorial approach to measuring sleep, as the use of a subjective measurement alone did not show a relationship with physical health-related quality of life. While subjective and objective sleep measurements share commonalities and are conceptually equivalent, previous research has demonstrated inconsistencies between the measurements $[15,28,52-55]$. In line with previous research, our study found that subjective and objective sleep measurements were either weakly or not significantly correlated [15,28,52-55]. Previous studies examining the agreement between objective and subjective sleep measurements have reported both under- and overestimations of sleep efficiency [28,52]. In a study of older adults, $54 \%$ of participants 
underestimated their sleep efficiency by $5 \%$ or more as compared with what was objectively observed by actigraphy, whereas $27 \%$ of participants overestimated their sleep efficiency by $5 \%$ or more [28]. Previous research has shown that individuals with insomnia tend to underestimate their sleep duration and overestimate their sleep latency [54] which results in an overall underestimation of sleep efficiency. The under and overestimating of various aspects of sleep highlight the need for objective sleep measurements to supplement subjective measurements.

\subsection{Strengths and Weaknesses}

The present study has some notable strengths. The study included a multifactorial approach to sleep, encompassing both subjective and objective sleep parameters. Secondly, the study involved working individuals rather than individuals from disease cohorts. Thus, the results from this study may be more generalizable to the general population than those arising from studies of specific clinical populations. Finally, to our knowledge, this is the first study that studied the effects of both objective and subjective sleep parameters and health-related quality of life in a working population in Singapore. This study, however, is not without its limitations. Firstly, although the study used standardised and validated questionnaires, self-report questionnaires are susceptible to recall and social desirability bias. Secondly, one-time reporting of sleep variables may not accurately reflect the dynamic changes in sleep pattern, and thus long term effects of quality and quantity of sleep on physical and mental health-related quality of life, in addition to not being able to establish temporality and causality. Thirdly, the PSQI elicits responses from participants that best reflect their sleep during the previous month. The eight-day period of actigraphic recording was, thus, not aligned over the same reporting period of the PSQI. Furthermore, current recommendations for improved actigraphic assessment of sleep quality call for 14-day recordings to account for day-to-day, as well as week-to-week, variability in sleep quality [56]. Additionally, almost $80 \%$ of study participants were men, as the industries are comprised mainly of positions taken up by men such as engineers, technicians, and traffic controllers. Therefore, results may not be as generalizable to the female population of workers in Singapore. Lastly, the SD scores of the mean PCS and MCS scores were quiet small and this may be the result of using 2009 U.S. norms for scoring PCS and MCS. Ideally PCS and MCS scoring should be generated based on norms from the local population, however, this option was unavailable to us at the time of the study.

\subsection{Future Directions}

For future research, it would be necessary to replicate this study with a larger sample, with workers from varying industries, and applying recruitment strategies to ensure a greater percentage of females are enrolled. As this study only included a single self-report instrument to assess sleep quality, additional self-report tools examining other aspects of sleep or specific disorders, for example, sleep apnea or insomnia, should be considered in future studies of the relationship between sleep and health-related quality of life. Sleep hygiene education in workplaces in Singapore has the potential to improve both the quality and quantity of worker's sleep and may also help improve worker's health-related quality of life, and therefore the effects should be studied in a longitudinal fashion. Furthermore, in a longitudinal design, the bidirectional relationship between sleep and health-related quality of life may be better understood.

Further study is needed to examine the workplace factors which may be contributing to sleep disturbances, the mechanistic pathways linking sleep disturbances and reduced health-related quality of life, as well as the impact of low health-related quality of life on work performance and productivity. These will better inform the allocation of resources for an increased outreach of educational efforts aimed at improving sleep health literacy and modifying workplace health policies to improve sleep health and quality of life among workers in Singapore. 


\section{Conclusions}

The application of subjective and objective sleep measurements in parallel identified a number of sleep parameters that were associated with health-related quality of life, even after adjusting for numerous confounders related to sociodemographic, health, lifestyle, and occupational factors. Subjectively reported sleep disturbances were associated with a lower physical health-related quality of life, whereas higher objective, but not subjective, sleep efficiency was associated with greater physical health-related quality of life. The PSQI domain "daytime dysfunction" was independently associated with a lower mental health-related quality of life. More research is necessary to explore the mechanistic pathways linking sleep impairment and reduced health-related quality of life, as well as the impact of health-related quality of life on work performance and productivity. Workplace health promotion planners should consider programmes that educate workers on better sleep hygiene practices in an effort to improve sleep quality and health-related quality of life.

Supplementary Materials: The following are available online at http://www.mdpi.com/1660-4601/16/21/4147/s1, Table S1: Characteristics of study participants (Health, lifestyle and occupational factors), Table S2: Univariate associations with Physical Component Summary (PCS) and Mental Component Summary (MCS), Table S3: Multivariable analysis for objective sleep parameters and Physical Component Summary, Table S4: Multivariable analysis for subjective sleep parameters and Physical Component Summary, Table S5: Multivariable analysis for objective sleep parameters and Mental Component Summary, Table S6: Multivariable analysis for subjective sleep parameters and Mental Component Summary.

Author Contributions: Conceptualization, G.D. and J.C.; methodology, G.D., A.C.T. and T.-Q.T.; formal analysis, G.D., Y.Z.; investigation, G.D., C.-K.S., G.C. and J.C.; data curation, G.D.; writing-original draft preparation, G.D. and A.C.T.; writing-review and editing, G.D., A.C.T., A.P.C., Y.Z., K.L.C., T.-Q.T., Y.R., C.-K.S., G.C., H.d.V. and J.C.; supervision, J.C., K.L.C. and H.d.V.; project administration, G.D.; funding acquisition, C.-K.S., G.C. and J.C.

Funding: This research is supported in part by the Singapore Ministry of National Development and the National Research Foundation, Prime Minister's Office under the Land and Liveability National Innovation Challenge (L2 NIC) Research Programme (L2 NIC Award No L2 NIC FP1-2013-2). Any opinions, findings, and conclusions or recommendations expressed in this material are those of the author(s) and do not reflect the views of the Singapore Ministry of National Development and National Research Foundation, Prime Minister's Office, Singapore.

Acknowledgments: We thank the participants who took time off their busy schedules to participate in our study.

Conflicts of Interest: The authors declare no conflict of interest. The funders had no role in the design of the study; in the collection, analyses, or interpretation of data; in the writing of the manuscript, or in the decision to publish the results".

\section{References}

1. Brown, R.E.; Basheer, R.; McKenna, J.T.; Strecker, R.E.; McCarley, R.W. Control of sleep and wakefulness. Physiol. Rev. 2012, 92, 1087-1187. [CrossRef] [PubMed]

2. Tononi, G.; Cirelli, C. Sleep and the price of plasticity: From synaptic and cellular homeostasis to memory consolidation and integration. Neuron 2014, 81, 12-34. [CrossRef] [PubMed]

3. Medic, G.; Wille, M.; Hemels, M.E. Short- and long-term health consequences of sleep disruption. Nat. Sci Sleep 2017, 9, 151-161. [CrossRef] [PubMed]

4. Buysse, D.J. Sleep Health: Can We Define It? Does It Matter? Sleep 2014, 37, 9-17. [CrossRef]

5. Walch, O.J.; Cochran, A.; Forger, D.B. A global quantification of "normal" sleep schedules using smartphone data. Sci. Adv. 2016, 2, e1501705. [CrossRef]

6. Manpower Research \& Statistics Department, MOM. Labour Market Survey. Available online: https: //stats.mom.gov.sg/Pages/Hours-Worked-Summary-Table.aspx (accessed on 1 July 2019).

7. U.S. Bureau of Labor Statistics-Average Weekly Hours and Overtime of All Employees on Private Nonfarm Payrolls by Industry Sector, Seasonally Adjusted. Available online: https://www.bls.gov/news.release/empsit. t18.htm (accessed on 1 July 2019).

8. Falchi, F.; Cinzano, P.; Duriscoe, D.; Kyba, C.C.M.; Elvidge, C.D.; Baugh, K.; Portnov, B.A.; Rybnikova, N.A.; Furgoni, R. The new world atlas of artificial night sky brightness. Sci. Adv. 2016, 2, e1600377. [CrossRef]

9. Lim, S.M.; Chia, S.E. The prevalence of fatigue and associated health and safety risk factors among taxi drivers in Singapore. Singap. Med. J. 2015, 56, 92-97. [CrossRef] 
10. Grandner, M.A. Sleep, Health, and Society. Sleep Med. Clin. 2017, 12, 1-22. [CrossRef]

11. Leow, M.K.-S.; Griva, K.; Choo, R.; Wee, H.-L.; Thumboo, J.; Tai, E.S.; Newman, S. Determinants of Health-Related Quality of Life (HRQoL) in the Multiethnic Singapore Population-A National Cohort Study. PLOS ONE 2013, 8, e67138. [CrossRef]

12. Kong, K.H.; Yang, S.Y. Health-related quality of life among chronic stroke survivors attending a rehabilitation clinic. Singap. Med. J. 2006, 47, 213-218.

13. Luo, N.; Calvin Soon-Leng, F.; Thumboo, J.; Shu-Chuen, L. Factors Influencing Health-Related Quality of Life of Asians with Anxiety Disorders in Singapore. Qual. Life Res. 2004, 13, 557-565. [CrossRef] [PubMed]

14. Buysse, D.J.; Reynolds, C.F., 3rd; Monk, T.H.; Berman, S.R.; Kupfer, D.J. The Pittsburgh Sleep Quality Index: A new instrument for psychiatric practice and research. J. Psychiatr. Res. 1989, 28, 193-213. [CrossRef]

15. Ancoli-Israel, S.; Cole, R.; Alessi, C.; Chambers, M.; Moorcroft, W.; Pollak, C.P. The Role of Actigraphy in the Study of Sleep and Circadian Rhythms. Sleep 2003, 26, 342-392. [CrossRef] [PubMed]

16. Littner, M.; Kushida, C.A.; Anderson, W.M.; Bailey, D.; Berry, R.B.; Davila, D.G.; Hirshkowitz, M.; Kapen, S.; Kramer, M.; Loube, D.; et al. Practice parameters for the role of actigraphy in the study of sleep and circadian rhythms: An update for 2002. Sleep 2003, 26, 337-341. [CrossRef] [PubMed]

17. Lee, M.; Choh, A.C.; Demerath, E.W.; Knutson, K.L.; Duren, D.L.; Sherwood, R.J.; Sun, S.S.; Chumlea, W.M.C.; Towne, B.; Siervogel, R.M.; et al. Sleep disturbance in relation to health-related quality of life in adults: The Fels Longitudinal Study. J. Nutr. Health Aging 2009, 13, 576-583. [CrossRef] [PubMed]

18. Darchia, N.; Oniani, N.; Sakhelashvili, I.; Supatashvili, M.; Basishvili, T.; Eliozishvili, M.; Maisuradze, L.; Cervena, K. Relationship between Sleep Disorders and Health Related Quality of Life-Results from the Georgia SOMNUS Study. Int J. Environ. Res. Public Health 2018, 15, 1588. [CrossRef]

19. Strine, T.W.; Chapman, D.P. Associations of frequent sleep insufficiency with health-related quality of life and health behaviors. Sleep Med. 2005, 6, 23-27. [CrossRef]

20. Lima, M.G.; Barros, M.B.D.A.; Alves, M.C.G.P. Sleep duration and health status self-assessment (SF-36) in the elderly: A population-based study (ISA-Camp 2008). Cadernos Saude Publica 2012, 28, 1674-1684. [CrossRef]

21. Abell, J.G.; Shipley, M.J.; Ferrie, J.E.; Kivimäki, M.; Kumari, M. Association of chronic insomnia symptoms and recurrent extreme sleep duration over 10 years with well-being in older adults: A cohort study. BMJ Open 2016, 6, e009501. [CrossRef]

22. Aritake, S.; Asaoka, S.; Kagimura, T.; Shimura, A.; Futenma, K.; Komada, Y.; Inoue, Y. Internet-Based Survey of Factors Associated with Subjective Feeling of Insomnia, Depression, and Low Health-Related Quality of Life Among Japanese Adults with Sleep Difficulty. Int. J. Behav. Med. 2015, 22, 233-238. [CrossRef]

23. Hammersen, F.; Lewin, P.; Gebauer, J.; Kreitschmann-Andermahr, I.; Brabant, G.; Katalinic, A.; Waldmann, A. Sleep quality and health-related quality of life among long-term survivors of (non-) Hodgkin lymphoma in Germany. PLoS ONE 2017, 12, e0187673. [CrossRef] [PubMed]

24. Komada, Y.; Nomura, T.; Kusumi, M.; Nakashima, K.; Okajima, I.; Sasai, T.; Inoue, Y. A two-year follow-up study on the symptoms of sleep disturbances/insomnia and their effects on daytime functioning. Sleep Med. 2012, 13, 1115-1121. [CrossRef] [PubMed]

25. Schubert, C.R.; Cruickshanks, K.J.; Dalton, D.S.; Klein, B.E.; Klein, R.; Nondahl, D.M. Prevalence of sleep problems and quality of life in an older population. Sleep 2002, 25, 889-893. [PubMed]

26. Rafie, C.; Ning, Y.; Wang, A.; Gao, X.; Houlihan, R. Impact of physical activity and sleep quality on quality of life of rural residents with and without a history of cancer: Findings of the Day and Night Study. Cancer Manag. Res. 2018, 10, 5525-5535. [CrossRef] [PubMed]

27. Lockley, S.W.; Skene, D.J.; Arendt, J. Comparison between subjective and actigraphic measurement of sleep and sleep rhythms. J. Sleep Res. 1999, 8, 175-183. [CrossRef] [PubMed]

28. Hughes, J.M.; Song, Y.; Fung, C.H.; Dzierzewski, J.M.; Mitchell, M.N.; Jouldjian, S.; Josephson, K.R.; Alessi, C.A.; Martin, J.L. Measuring Sleep in Vulnerable Older Adults: A Comparison of Subjective and Objective Sleep Measures. Clin. Gerontol. 2018, 41, 145-157. [CrossRef] [PubMed]

29. Kay, D.B.; Buysse, D.J.; Germain, A.; Hall, M.; Monk, T.H. Subjective-objective sleep discrepancy among older adults: Associations with insomnia diagnosis and insomnia treatment. J. Sleep Res. 2015, 24, 32-39. [CrossRef]

30. Iliescu, E.A.; Coo, H.; McMurray, M.H.; Meers, C.L.; Quinn, M.M.; Singer, M.A.; Hopman, W.M. Quality of sleep and health-related quality of life in haemodialysis patients. Nephrol. Dial. Transpl. 2003, 18, 126-132. [CrossRef] 
31. Liu, H.-X.; Lin, J.; Lin, X.-H.; Wallace, L.; Teng, S.; Zhang, S.-P.; Hao, Y.-F. Quality of sleep and health-related quality of life in renal transplant recipients. Int. J. Clin. Exp. Med. 2015, 8, 16191-16198.

32. Parvan, K.; Lakdizaji, S.; Roshangar, F.; Mostofi, M. Quality of sleep and its relationship to quality of life in hemodialysis patients. J. Caring Sci. 2013, 2, 295-304.

33. Bais, B.; Lindeboom, R.; van Ravesteyn, L.; Tulen, J.; Hoogendijk, W.; Lambregtse-van den Berg, M.; Kamperman, A. The Impact of Objective and Subjective Sleep Parameters on Depressive Symptoms during Pregnancy in Women with a Mental Disorder: An Explorative Study. Int. J. Environ. Res. Public Health 2019, 16, 1587. [CrossRef] [PubMed]

34. Dunleavy, G.; Sathish, T.; Nazeha, N.; Soljak, M.; Visvalingam, N.; Roberts, A.C.; Yap, H.S.; Bajpai, R.; Thach, T.Q.; Tonin, A.C.; et al. Health Effects of Underground Workplaces (HEUW) cohort: Study design and baseline characteristics. Epidemiol. Health 2019, e2019036. [CrossRef]

35. Ware, J.E., Jr.; Sherbourne, C.D. The MOS 36-item short-form health survey (SF-36). I. Conceptual framework and item selection. Med. Care 1992, 30, 473-483. [CrossRef] [PubMed]

36. Mollayeva, T.; Thurairajah, P.; Burton, K.; Mollayeva, S.; Shapiro, C.M.; Colantonio, A. The Pittsburgh sleep quality index as a screening tool for sleep dysfunction in clinical and non-clinical samples: A systematic review and meta-analysis. Sleep Med. Rev. 2016, 25, 52-73. [CrossRef]

37. Dean, D.A., 2nd; Goldberger, A.L.; Mueller, R.; Kim, M.; Rueschman, M.; Mobley, D.; Sahoo, S.S.; Jayapandian, C.P.; Cui, L.; Morrical, M.G.; et al. Scaling Up Scientific Discovery in Sleep Medicine: The National Sleep Research Resource. Sleep 2016, 39, 1151-1164. [CrossRef]

38. Rosengren, A.; Hawken, S.; Ounpuu, S.; Sliwa, K.; Zubaid, M.; Almahmeed, W.A.; Blackett, K.N.; Sitthi-amorn, C.; Sato, H.; Yusuf, S. Association of psychosocial risk factors with risk of acute myocardial infarction in 11119 cases and 13648 controls from 52 countries (the INTERHEART study): Case-control study. Lancet 2004, 364, 953-962. [CrossRef]

39. Koh, H.W.L.; Lim, R.B.T.; Chia, K.S.; Lim, W.Y. The Pittsburgh Sleep Quality Index in a multi-ethnic Asian population contains a three-factor structure. Sleep Breath 2015, 19, 1147-1154. [CrossRef]

40. Shum, A.; Taylor, B.J.; Thayala, J.; Chan, M.F. The effects of sedative music on sleep quality of older community-dwelling adults in Singapore. Complement. Ther. Med. 2014, 22, 49-56. [CrossRef]

41. Chiang, G.S.H.; Sim, B.L.H.; Lee, J.J.M.; Quah, J.H.M. Determinants of poor sleep quality in elderly patients with diabetes mellitus, hyperlipidemia and hypertension in Singapore. Prim. Health Care Res. Dev. 2018, 19, 610-615. [CrossRef]

42. Liguori, C.; Mercuri, N.B.; Izzi, F.; Romigi, A.; Cordella, A.; Sancesario, G.; Placidi, F. Obstructive Sleep Apnea is Associated with Early but Possibly Modifiable Alzheimer's Disease Biomarkers Changes. Sleep 2017, 40. [CrossRef]

43. Hall, M.H.; Okun, M.L.; Sowers, M.; Matthews, K.A.; Kravitz, H.M.; Hardin, K.; Buysse, D.J.; Bromberger, J.T.; Owens, J.F.; Karpov, I.; et al. Sleep Is Associated with the Metabolic Syndrome in a Multi-Ethnic Cohort of Midlife Women: The SWAN Sleep Study. Sleep 2012, 35, 783-790. [CrossRef] [PubMed]

44. Dew, M.A.; Hoch, C.C.; Buysse, D.J.; Monk, T.H.; Begley, A.E.; Houck, P.R.; Hall, M.; Kupfer, D.J.; Reynolds, C.F., 3rd. Healthy older adults' sleep predicts all-cause mortality at 4 to 19 years of follow-up. Psychosom. Med. 2003, 65, 63-73. [CrossRef] [PubMed]

45. Silva, G.E.; An, M.-W.; Goodwin, J.L.; Shahar, E.; Redline, S.; Resnick, H.; Baldwin, C.M.; Quan, S.F. Longitudinal evaluation of sleep-disordered breathing and sleep symptoms with change in quality of life: The Sleep Heart Health Study (SHHS). Sleep 2009, 32, 1049-1057. [CrossRef] [PubMed]

46. Adams, R.J.; Appleton, S.L.; Vakulin, A.; Lang, C.; Martin, S.A.; Taylor, A.W.; McEvoy, R.D.; Antic, N.A.; Catcheside, P.G.; Wittert, G.A. Association of daytime sleepiness with obstructive sleep apnoea and comorbidities varies by sleepiness definition in a population cohort of men. Respirology 2016, 21, 1314-1321. [CrossRef] [PubMed]

47. Hirshkowitz, M.; Whiton, K.; Albert, S.M.; Alessi, C.; Bruni, O.; DonCarlos, L.; Hazen, N.; Herman, J.; Katz, E.S.; Kheirandish-Gozal, L.; et al. National Sleep Foundation's sleep time duration recommendations: Methodology and results summary. Sleep Health 2015, 1, 40-43. [CrossRef]

48. Abbott, S.M.; Malkani, R.G.; Zee, P.C. Circadian disruption and human health: A bidirectional relationship. Eur. J. Neurosci. 2018. [CrossRef]

49. Lee, S.; Buxton, O.M.; Andel, R.; Almeida, D.M. Bidirectional associations of sleep with cognitive interference in employees' work days. Sleep Health 2019, 5, 298-308. [CrossRef] 
50. Stein, M.B.; Belik, S.L.; Jacobi, F.; Sareen, J. Impairment associated with sleep problems in the community: Relationship to physical and mental health comorbidity. Psychosom. Med. 2008, 70, 913-919. [CrossRef]

51. Van Dyk, T.R.; Thompson, R.W.; Nelson, T.D. Daily Bidirectional Relationships Between Sleep and Mental Health Symptoms in Youth with Emotional and Behavioral Problems. J. Pediatr. Psychol. 2016, 41, 983-992. [CrossRef]

52. Bei, B.; Milgrom, J.; Ericksen, J.; Trinder, J. Subjective Perception of Sleep, but not its Objective Quality, is Associated with Immediate Postpartum Mood Disturbances in Healthy Women. Sleep 2010, 33, 531-538. [CrossRef]

53. Baillet, M.; Cosin, C.; Schweitzer, P.; Pérès, K.; Catheline, G.; Swendsen, J.; Mayo, W. Mood Influences the Concordance of Subjective and Objective Measures of Sleep Duration in Older Adults. Front. Aging Neurosci. 2016, 8, 181. [CrossRef] [PubMed]

54. Frankel, B.L.; Coursey, R.D.; Buchbinder, R.; Snyder, F. Recorded and reported sleep in chronic primary insomnia. Arch. Gen. Psychiatry 1976, 33, 615-623. [CrossRef] [PubMed]

55. Van Den Berg, J.F.; Van Rooij, F.J.; Vos, H.; Tulen, J.H.; Hofman, A.; Miedema, H.M.; Neven, A.K.; Tiemeier, H. Disagreement between subjective and actigraphic measures of sleep duration in a population-based study of elderly persons. J. Sleep Res. 2008, 17, 295-302. [CrossRef] [PubMed]

56. Van Someren, E. Improving actigraphic sleep estimates in insomnia and dementia: How many nights? J. Sleep Res. 2007, 16, 269-275. [CrossRef] [PubMed]

(C) 2019 by the authors. Licensee MDPI, Basel, Switzerland. This article is an open access article distributed under the terms and conditions of the Creative Commons Attribution (CC BY) license (http://creativecommons.org/licenses/by/4.0/). 